Cite this: J. Mater. Chem B, 2013, 1 5456

Received 20th March 2013 Accepted 8th August 2013 DOI: $10.1039 / c 3 t b 20388 c$ www.rsc.org/MaterialsB

\section{Biologically triggered liberation of sub-micron particles from alginate microcapsules $\uparrow$}

\author{
Jitka Čejková, Petra Haufová, Damian Gorný, Jaroslav Hanuš and František Štěpánek* \\ A new method for triggering the burst liberation of encapsulated sub-micron particles from carrier \\ particles using embedded microorganisms has been developed. Triggering mechanisms such as those \\ based on chemical, light, thermal, or magnetic stimuli are known, but man-made particles are not yet \\ able to replicate the concept of "dormancy" found in biological systems in the form of spores or seeds \\ that survive in an inactive state and start to grow only once favourable environmental conditions are \\ encountered. An engineered particle system that mimics this property by embedding viable yeast cells \\ into synthetically made alginate microcapsules is reported in the present work. Cell growth and division \\ is used as a triggering mechanism for stimuli-responsive release of the encapsulated content. The hybrid \\ living/artificial capsules were formed by an inkjet printing process and the mechanism of biologically \\ triggered release was shown using fluorescently labelled liposomes.
}

\section{Introduction}

The controlled release of active substances (e.g. drugs, fragrances, probiotics, nutrient preservatives) from man-made capsules is of considerable interest in many fields of science and technology. ${ }^{1}$ A variety of chemical and physical methods have been developed to release the capsule contents, $c f$. e.g. a review by Esser-Kahn et al. ${ }^{2}$ The release triggers are frequently based on mechanical, chemical, electrical, thermal, photo, or magnetic stimuli. While these stimuli are well-defined in terms of their timing and the threshold value of a physical quantity that characterises each stimulus (e.g. voltage, temperature, concentration, etc.), release triggers occurring in nature are often more complex and comprise the simultaneous occurrence of several stimuli - for example, the combination of specific temperature, humidity, light conditions and nutrients is necessary for seed germination, etc.

The use of actual living microorganisms or their spores offers itself as a possible way of achieving such complex triggers even in synthetically made particles. To achieve this, a necessary condition is the ability to fabricate composite microcapsules that incorporate the chosen microorganism or its spores, and to ensure their viability. In the specific case of yeast cell encapsulation into alginate gel, currently available encapsulation techniques include microfluidic approaches, ${ }^{3}$ electrostatic droplet generation, ${ }^{4}$ spinning disk atomization ${ }^{5}$ or simply dropping an alginate solution into $\mathrm{CaCl}_{2}$ using a syringe. ${ }^{6,7}$ At

Institute of Chemical Technology Prague, Department of Chemical Engineering, Technicka 5, Prague 6, 166 28, Czech Republic. E-mail: frantisek.stepanek@vscht.cz; Tel: +420220443236

$\dagger$ Electronic supplementary information (ESI) available. See DOI: $10.1039 / \mathrm{c} 3 \mathrm{tb} 20388 \mathrm{c}$ present, the main reasons for microbial cell encapsulation into various matrixes include cell protection from negative environmental influences such as shear forces, phagocytosis or digestion. Immobilized microbial cells also can be handled more easily and separated from a solution. ${ }^{8}$

In most current applications of immobilised cells, microcapsule rupture due to internal pressure of proliferating cells is usually not of interest. In the case of cell-based biosensor applications, the cell division and capsule disintegration would be outright undesirable. Cases where the capsules rupture due to $\mathrm{CO}_{2}$ formation when they are placed in a growth medium have been reported ${ }^{9}$ and the surfactant Tween- 20 has been used to improve the permeability of the capsule for easier $\mathrm{CO}_{2}$ liberation. ${ }^{6}$ A silica coating can also enhance the mechanical resistance of capsules. ${ }^{10}$ A case where the cell division is a wanted process has been reported by Hamad et al. ${ }^{\mathbf{1 1}}$ who fabricated composite multi-cell/shellac microcapsules that contained living yeast cells. Cell release from the microcapsule after incubation in a cultivation medium has been demonstrated. However, to the best of our knowledge, the use of cell growth as a trigger for the release of another active substance also embedded within the carrier particle has not been reported in the literature as yet.

The present paper describes the preparation of hybrid alginate microcapsules capable of releasing an encapsulated payload - fluorescently labelled liposomes in this case - as a result of rupture caused by proliferating cells. The schematic structure and mechanism of action of the microcapsules is shown in Fig. 1. Under unfavourable conditions (absence of nutrients, low temperature) cell division does not occur and the microcapsules are stable in aqueous medium for extended periods of time without disintegration or release of their 


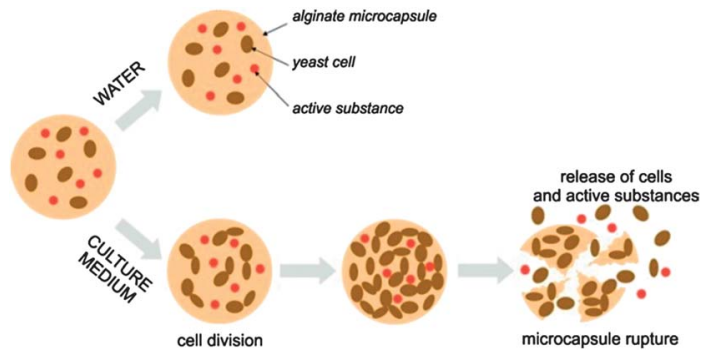

Fig. 1 Schematic principle of microcapsule rupture and liberation of an active substance into the environment caused by yeast cell growth in the culture medium.

content. Once the microcapsules encounter favourable conditions (presence of nutrients, higher temperature), cell division and growth causes an eventual rupture of the alginate capsule and release of the embedded liposomes. Additionally, magnetic iron oxide nanoparticles were also embedded within the composite microcapsules to facilitate their manipulation and separation by a magnetic field. Liposomes loaded with fluorescein represent a model "active" particulate substance that is to be liberated from the composite microcapsules. Calcium alginate hydrogel was chosen as the matrix material in which the three types of internal components (yeast cells, liposomes, magnetic nanoparticles) were embedded due to its ability to form microcapsules by means of an inkjet printing process, which has been demonstrated in our previous work..$^{12,13}$

\section{Materials and methods}

\subsection{Materials}

Sodium alginate, calcium chloride $\left(\mathrm{CaCl}_{2}\right)$, fluorescein diacetate (FDA), yeast extract and glucose were purchased from SigmaAldrich. Instant yeast cells (Labeta a.s., Czech Republic) were suspended in deionised water in various concentrations $(1 \mathrm{mg}$ of dry powder corresponds to $3 \times 10^{7}$ cells). Hydrophilic iron oxide nanoparticles were prepared according to a synthesis method described in ref. 14. Fluorescently labelled liposomes (molar ratio of DPPC : cholesterol was $2: 1$ ) were synthesized in the same way as described in ref. 15. Deionised water was produced using an ionex filter (Aqual 25).

\subsection{Microcapsule formation}

All microcapsules were produced by inkjet printing. ${ }^{16} \mathrm{~A}$ piezoelectric drop-on-demand print-head type M5-ABP-01-80-6MX supplied by Microfab, Inc. (Plano, Texas, USA) was used, coupled with a control unit type JetDrive III and a pressure controller type CT-PT-01 also supplied by Microfab, Inc. $2 \mathrm{ml}$ of aqueous solutions of $2 \%(\mathrm{w} / \mathrm{w})$ sodium alginate and $2 \mathrm{ml}$ of aqueous suspension of yeast cells were mixed and printed into approximately $50 \mathrm{ml}$ of aqueous solution of $2 \%(\mathrm{w} / \mathrm{w}) \mathrm{CaCl}_{2}$ where a rapid ionic cross-linking of the microdroplets occurred. The receiving $\mathrm{CaCl}_{2}$ solution was constantly agitated to avoid coalescence of microdroplets after impact. To prepare magnetic microcapsules, one half of the cell suspension was replaced by citrate-stabilized iron oxide nanoparticle dispersion in water (15 $\mathrm{mg} \mathrm{ml}{ }^{-1}$ ). The solution for printing of magnetic capsules containing liposomes was obtained from a sodium alginate solution, the cell suspension, the iron oxide nanoparticle solution and a liposome solution in the volume ratio $4: 1: 1: 2$. Cross-linked calcium alginate microcapsules were separated from the $\mathrm{CaCl}_{2}$ solution by using a filter or magnet and suspended in deionized water in which they were stored at room temperature until further use. In this state the composite microcapsules were stable for up to 4 months without any significant loss of yeast cell viability.

\subsection{Microcapsule characterization}

The microcapsules were characterized by means of an inverted optical microscope (Olympus CK40) and a laser scanning confocal microscope - LSCM (Olympus Fluoview FV1000). The particle size was evaluated by laser diffraction (Horiba Partica LA 950/V2). The viability of yeast cells was confirmed by using the standard fluorescein diacetate solution method.

\subsection{Yeast cell division and microcapsule disruption study}

For a study of the cell division and disintegration of microcapsules, the composite microcapsules were placed into a Petri dish containing a culture medium (consisting of glucose at a concentration of $10 \mathrm{~g} \mathrm{l}^{-1}$ and yeast extract at a concentration of $5 \mathrm{~g} \mathrm{l}^{-1}$ ) and monitored using an optical microscope for 24 hours. The cell growth curves were measured by means of a visible spectrophotometer (Specord 205 BU, Analytik Jena, Germany); the wavelength used for the measurement of optical density was $600 \mathrm{~nm}$ (OD 600).

\section{Results and discussion}

\subsection{Microcapsule characterization}

The drop-on-demand inkjet technology was used for the formation of calcium alginate microcapsules with embedded yeast cells by ejecting droplets of a sodium alginate precursor into a pool of calcium chloride solution. The shape of the formed microcapsules was mostly spherical, however, some of them were distorted (flattened) due to droplet deformation upon landing into the $\mathrm{CaCl}_{2}$ solution. ${ }^{5}$ Fig. $2(\mathrm{~A})$ and (B) show the composite alginate microcapsules with two different cell concentrations; both spherical and flattened capsules are evident irrespective of the cell concentration used.
(A)
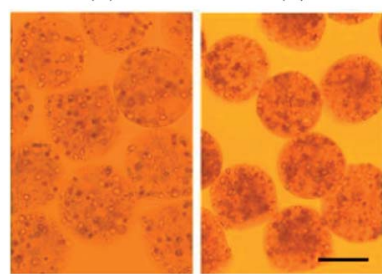

(C)

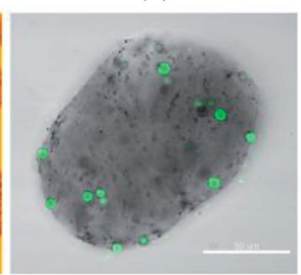

Fig. 2 Magnetic alginate microcapsules with various cell concentrations. Optical microscopy images: (A) $2.25 \times 10^{8}$ cells per $\mathrm{ml}$ and (B) $3.75 \times 10^{8}$ cells per $\mathrm{ml}$. (C) Laser scanning confocal microscopy image (single slice in the $x-y$ plane) of a 1 month old magnetic alginate microcapsule with $2.25 \times 10^{8}$ cells per $\mathrm{ml}$ labelled by FDA. Scale bars represent $50 \mu \mathrm{m}$. 
The mean size of freshly precipitated capsules measured in $\mathrm{CaCl}_{2}$ solution by means of static light scattering was $61 \mu \mathrm{m}$; in water the mean size increased to $79 \mu \mathrm{m}$ due to swelling of calcium alginate. This is provoked by the relaxation of the polymer network at the presence of osmotic pressure. Swelling of the calcium alginate beads in water occurs until the osmotic pressure equals the forces of the cross-linking bonds that maintain the structure of the polymer network. ${ }^{17}$

The viability of yeast cells in the composite microcapsules was confirmed by using fluorescein diacetate (FDA).$^{18}$ This colourless compound exhibits no fluorescence, however, it is known that it diffuses through the cell membrane and living cells are able to hydrolyse it by their enzymatic apparatus and transform FDA into fluorescein. Typically, $2 \mathrm{ml}$ of microcapsule suspension were incubated with a few droplets of FDA in acetone for 20 minutes, then washed and observed under a LSCM. By this test it was proven that the cells are able to retain their viability during the inkjet printing process. Fig. 2(C) shows a microcapsule after one month of its fabrication and storage in water that was incubated with FDA. The green spots correspond to living cells, which confirms that the cell viability is preserved for many weeks. The viability tests also excluded that the magnetic iron oxide nanoparticles have a harmful effect on yeast cells. Viable yeast cells clearly exist in the composite microcapsules in the presence of inorganic nanoparticles.

\subsection{Yeast cell growth in microcapsules}

The hybrid microcapsules with embedded yeast cells were stored in water for a few weeks and no microcapsule changes or cell division in capsules was observed. Radical changes occurred only after incubation with a growth medium containing yeast extract and glucose. For the preparation of the culture medium we have used water instead of phosphate buffer, which is normally used, due to the fact that the phosphate buffer would precipitate calcium ions and reverse the alginate crosslinking. ${ }^{19}$

To observe the division of encapsulated yeast cells, composite microcapsules were suspended in a Petri dish with the growth medium and placed under a microscope at laboratory temperature $\left(\sim 25^{\circ} \mathrm{C}\right)$. Images in 1 minute intervals were grabbed for at least 24 hours and compiled into a movie (see ESI S1 $\dagger$ ). A series of experiments starting from capsules containing different initial cell concentrations with and without magnetic nanoparticles were performed. Typical results are summarized in Fig. 3 and 4 for microcapsules containing iron oxide nanoparticles and yeast cells at a concentration of $3.75 \times$ $10^{8}$ cells per ml.

Fig. 3 shows the evolution of microcapsule structure in time under static conditions in a Petri dish with a cultivation medium. During inkjet fabrication of the microcapsules, no cell loss was observed and the cell number per microcapsule was therefore determined by the cell concentration in the initial alginate solution. Time $t=0 \mathrm{~h}$ corresponds to the moment of placing microcapsules into the cultivation medium. About two hours after incubation, first buds on the cells appeared but no extensive budding was observed yet. Around the time $t=7 \mathrm{~h}$, the cell division was highly developed and around $t=15 \mathrm{~h}$ the alginate microcapsules were almost full of cells. At time $t=18 \mathrm{~h}$ the first ruptures of the composite microcapsules started. Due to the extensive cell division all microcapsules eventually disintegrated and essentially no original round microcapsules were evident at time $t=24 \mathrm{~h}$. Only clusters of yeast cells were present in the Petri dish.

The growth kinetics was also evaluated by using visible spectrophotometry, when the growth curves of cells in alginate

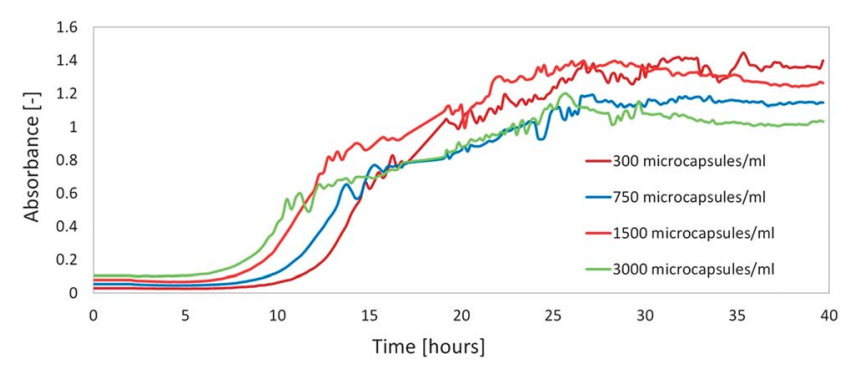

Fig. 4 Growth curves of yeast cells in the microcapsules (initial concentration $3.75 \times 10^{8}$ cells per $\mathrm{ml}$ ) for various concentrations of microcapsules in the growth medium. The curves correspond to different microcapsule dilutions as indicated in the legend.
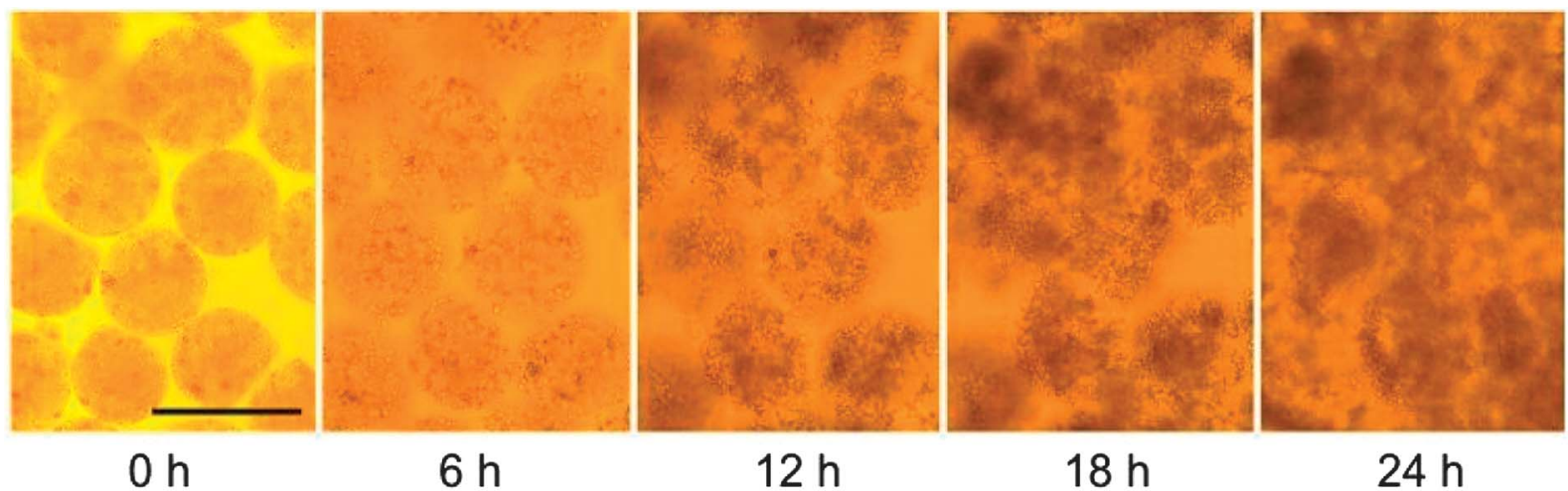

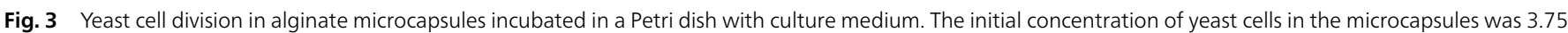
$\times 10^{8}$ cells per $\mathrm{ml}$. Scale bar represents $100 \mu \mathrm{m}$. 
microcapsules were measured as a function of optical density at the wavelength of $600 \mathrm{~nm}$. These two types of experiments differ in conditions. Observation by microscope was under static conditions without any mixing whereas microcapsules in the spectrophotometer cuvettes were intensively stirred using a magnetic stirrer in order to keep them suspended. Mixing might affect the microcapsule disintegration, therefore the capsules in cuvettes disintegrate somewhat earlier than on the Petri dishes.

Fig. 4 shows the changes in optical density of microcapsule suspension at $600 \mathrm{~nm}$. During the first few hours no changes were evident, because yeast cells were still in the lag phase of their growth, they adapted themselves to the growth conditions and rarely divided. At about $t=7 \mathrm{~h}$, the cells entered into the exponential growth phase and at about $t=12 \mathrm{~h}$ since the addition of nutrients the microcapsules ruptured. Despite mixing, the measured absorbance started to fluctuate at this stage. Nevertheless, a continuous increase of absorbance is evident until approximately $t=24 \mathrm{~h}$, which indicates continuing cell division. After the time $t=24 \mathrm{~h}$, the absorbance no longer increased as the cells entered into the stationary phase due to lack of nutrients.

To confirm that the disruption of alginate microcapsules was caused solely by the growth of encapsulated yeast cells, a blind experiment was performed. When alginate microcapsules without yeast cells were prepared and placed into the growth medium, no decomposition of the microcapsules was observed. Such a blind experiment excluded the growth medium as a possible factor causing the microcapsule rupture and confirmed the role of the dividing cells. To exclude the effect of yeast cells and the presence of their metabolites on the decomposition of alginate microcapsules, another blind experiment was performed. Alginate microparticles without embedded yeast were placed into a growth medium together with freely suspended yeast cells. Again, no microcapsule disintegration was observed, the cells have grown outside the microcapsules and did not affect the alginate integrity.

\subsection{Mimicking spore behaviour}

To demonstrate the concept of "artificial spores" or "artificial seeds", additional experiments with switching between favourable/unfavourable conditions and drying of microcapsules were performed. In biology, a spore is defined as a reproductive structure that is adapted for dispersion and survival for extended periods of time under unfavourable conditions. Once conditions are favourable, spores can develop into new organisms. The activators of such a transformation from a spore to a cell could be $e . g$. nutrients, temperature, $\mathrm{pH}$, or combination of these parameters. The interesting property of this transformation is that once the conditions are suitable for germination, the spores enter a lag phase and activate specific genes that trigger signal pathways leading to swelling and cell emergence. Once a spore has swollen, germination becomes irreversible, but during the lag phase activated spores can return to dormancy. ${ }^{20}$
The experiment mimicking the spore response to changes in conditions was performed in a spectrophotometer equipped with heating/cooling facility. The growth curves were measured and temperature changes applied. The experiment started under unfavourable conditions, where microcapsules were stored in water (without nutrients). The first change consisted of placing the capsules in the culture medium at a temperature of $30{ }^{\circ} \mathrm{C}$ (corresponding to time $t=0 \mathrm{~h}$ in the growth curves in Fig. 5). For the first few hours the yeast cells embedded in the microcapsules were still dormant. Once they entered the exponential phase of their growth, the temperature was rapidly decreased to $8{ }^{\circ} \mathrm{C}$. Such a temperature shock stopped the cell growth, resulting in a return to dormancy. During this time no change of optical density was observed, corresponding to no division of cells. When the temperature was increased back to $30{ }^{\circ} \mathrm{C}$, rapid cell growth was restarted and the next temperature decrease again caused an interruption of cell division. An example of three repetitions of heating/cooling is shown in Fig. 5(A). Fig. 5(B) shows the possibility to "freeze" the cell growth in microcapsule for almost two days and then restart growth by a return to favourable conditions.

\subsection{Cell growth triggered liberation of active substances from microcapsules}

As was shown above, composite microcapsules are stable in water, whereas after cultivation in a growth medium they are able to disintegrate. To demonstrate that capsule disintegration can be used for the release of a previously encapsulated payload, the liberation of fluorescently labelled liposomes was observed using a laser scanning confocal microscope. Fig. 6(A) shows the composite particles directly after their fabrication. The fluorescence signal is obtained only in the microcapsules, which confirms the presence of liposomes. In Fig. 6(B), the same microcapsules were imaged one day after fabrication and storage in pure water. Again, the fluorescence signal comes only from microcapsules, no liberation of liposomes from microcapsules occurred. On the other hand, Fig. 6(C) confirms that after cultivation of the microcapsules in a growth medium, the cells divide and cause the rupture of capsules, liberating the encapsulated substances into the surroundings. After one day

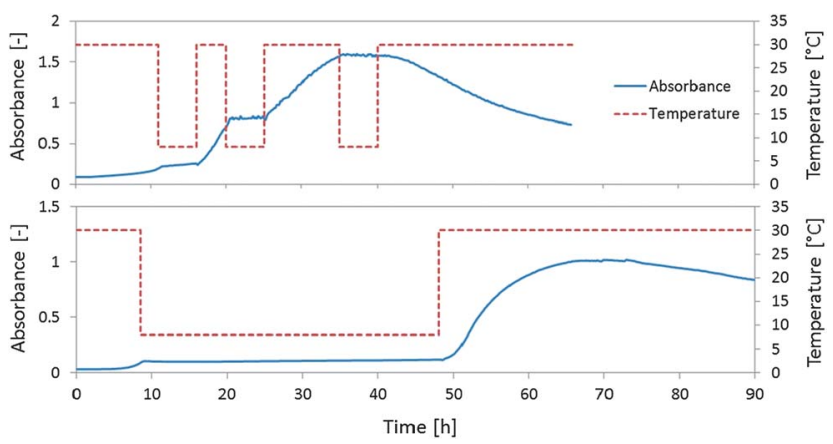

Fig. 5 Control of yeast growth by temperature modulation (interruption of growth by temperature decrease and resumption of growth by temperature increase). (A) Three interruptions of cell growth at times 11-16 h, 20-25 h, and 35-40 h. (B) One cell growth interruption for almost two days (8-48 h). 
(A)
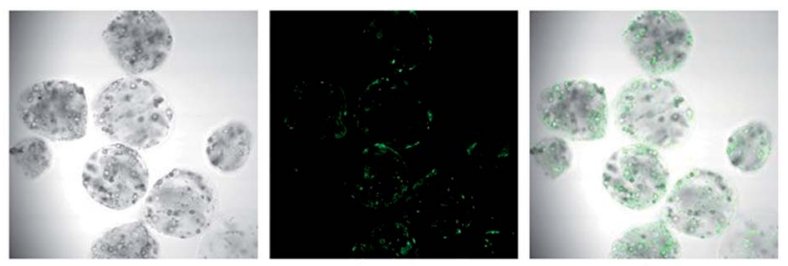

(B)
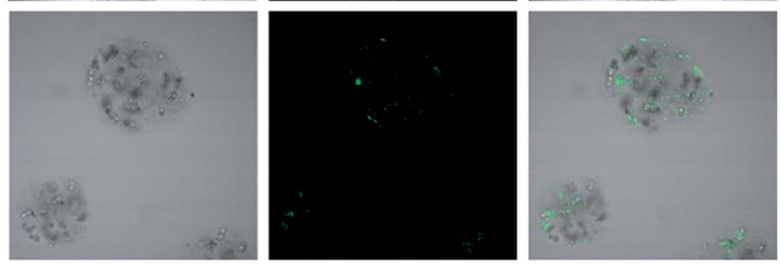

(C)
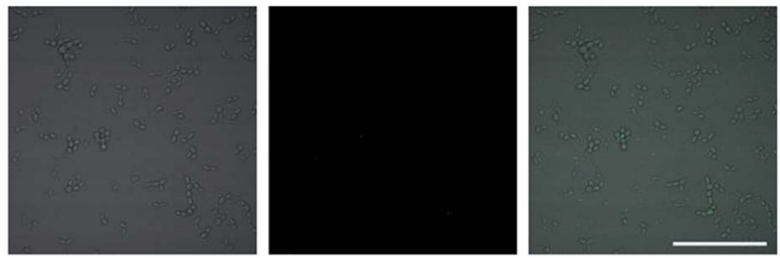

Fig. 6 Alginate microcapsules with fluorescently labelled liposomes. (A) Freshly prepared microcapsules in water. (B) Microcapsules after one day in water. (C) Disintegrated microcapsules after one day of incubation in a culture medium. (Left column - optical image, middle column - fluorescence signal, right column superposition of both signals. The scale bar represents $100 \mu \mathrm{m}$.)

of incubation in culture medium no compact microcapsules were present, only clusters of cells were evident and the fluorescence signal was detectable from the whole medium in a diluted way because the liposomes were released during the microcapsule disintegration. Microcapsule disintegration appears to be a "binary" event in the sense that partial rupture was rarely observed; once a microcapsule ruptured, it did so completely and the fluorescent signal from liberated liposomes was present uniformly in the background rather than clustered as would be the case if, e.g., some partially ruptured microcapsules prevailed in the system.

Having established that the liposome release by capsule rupture can be triggered by the cell division, the possibility to control the timing of rupture by the initial concentration of cells in the microcapsules was investigated. Three batches of microcapsules were prepared, with initial cell concentrations of 40-60 cells per particle, 10-20 cells per particle, and 3-6 cells per particle (obtained by dilutions of the initial yeast cell suspension in the alginate solution). The microcapsules were placed into a culture medium under favourable growth conditions and recorded in time. Once the microcapsules started to rupture, the percentage of fractured capsules as a function of time was evaluated by image analysis. The results are summarised in Fig. 7 and the obtained dependence of the fraction of ruptured particles as a function of time was fitted by a sigmoidal function of the form

$$
f(t)=1 /\left[1+\exp \left(-b\left(t-t_{\mathrm{m}}\right)\right)\right]
$$

where $t_{\mathrm{m}}$ is the mean rupture time and $b$ is a rate constant. The values of $b$ and $t_{\mathrm{m}}$ evaluated by non-linear regression of the data

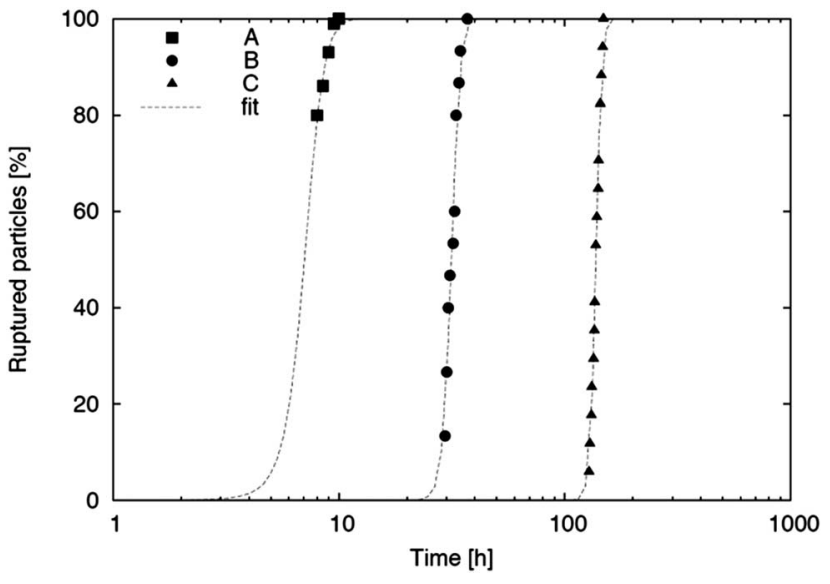

Fig. 7 Time of rupture of microparticles containing (A) 40-60, (B) 10-20 and (C) 3-6 cells per particle.

Table 1 Values of regression parameters from eqn (1) for three different cell concentrations in the microcapsules

\begin{tabular}{llr}
\hline Sample & $b\left[\mathrm{~h}^{-1}\right]$ & \multicolumn{1}{c}{$t_{\mathrm{m}}[\mathrm{h}]$} \\
\hline A & $1.38 \pm 0.27$ & $7.05 \pm 0.24$ \\
$\mathrm{~B}$ & $0.72 \pm 0.07$ & $31.46 \pm 0.13$ \\
$\mathrm{C}$ & $0.25 \pm 0.01$ & $137.15 \pm 0.18$
\end{tabular}

plotted in Fig. 7 are summarised in Table 1. As can be seen from both the figure and the table, there is a systematic dependence of the rupture time on the initial cell concentration, which offers the possibility to control the timing of the release.

\section{Conclusions}

Composite hydrogel microcapsules with embedded yeast cells that can act as a biological trigger for controlled opening of the microcapsules and locally liberate sub-micrometer objects have been fabricated. Additionally, magnetic nanoparticles were added into the microcapsules to enable manipulation by an external magnetic field. The composite capsules were stable in aqueous media for up to several months and retained their viability. Once favourable conditions were encountered - i.e. the presence of nutrients and a suitable temperature - the proliferation of yeast cells within the microcapsule leads to its eventual disintegration and the liberation of other embedded objects, namely fluorescently labelled liposomes. It has been shown that the biologically triggered release can be used not only for a oneoff rupture of the microcapsules, but also for a repeated interruption and 'restart' of the growth processes due to variations in external conditions. This behaviour contrasts with that of traditional man-made triggered release systems where irreversible processes tend to dominate. Systems based on biologically triggered release could find applications in fields such as controlled release of bactericides or fungicides, or the detection of potential food contamination due to inappropriate storage conditions. The principle of biologically triggered release can potentially be applied using other microorganisms than yeast cells, which 
would offer flexibility in the range of environmental conditions under which the composite capsules can operate, and also in the range of particle sizes (obviously, the initial particle must be large enough to contain at least several cells, as well as space for the payload that is to be liberated).

\section{Acknowledgements}

Financial support from the European Research Council (grant number 200580-Chobotix) and the European Union 7th Framework Program (Grant Agreement 318671-MICREAgents) is gratefully acknowledged.

\section{Notes and references}

1 X. Yan, J. Li and H. Möhwald, Adv. Mater., 2012, 24, 26632667.

2 A. P. Esser-Kahn, S. A. Odom, N. R. Sottos, S. R. White and J. S. Moore, Macromolecules, 2011, 44, 5539-5553.

3 C. J. Martinez, J. W. Kim, C. Ye, I. Ortiz, A. C. Rowat, M. Marquez and D. Weitz, Macromol. Biosci., 2012, 12, 946951.

4 V. A. Nedović, B. Obradović, I. Leskošek-Čukalović, O. Trifunović, R. Pešić and B. Bugarski, Process Biochem., 2001, 37, 17-22.

5 Y. Senuma, C. Lowe, Y. Zweifel, J. G. Hilborn and I. Marison, Biotechnol. Bioeng., 2000, 67, 616-622.

6 K. Koyama and M. Seki, J. Biosci. Bioeng., 2004, 97, 111-118.
7 H. N. Chang, G. H. Seong, I.-K. Yoo, J. K. Park and J.-H. Seo, Biotechnol. Bioeng., 1996, 51, 157-162.

8 J. K. Park and H. N. Chang, Biotechnol. Adv., 2000, 18, 303319.

9 S. Cheong, J. Park, B. Kim and H. Chang, Biotechnol. Tech., 1993, 7, 879-884.

10 T. Coradin, E. Mercey, L. Lisnard and J. Livage, Chem. Commun., 2001, 2496-2497.

11 S. A. Hamad, S. D. Stoyanov and V. N. Paunov, Soft Matter, 2012, 8, 5069-5077.

12 J. Dohnal and F. Štěpánek, Chem. Eng. Sci., 2011, 66, 38293835.

13 P. Haufová, J. Dohnal, J. Hanuš and F. Štěpánek, Colloids Surf., A, 2012, 410, 52-58.

14 V. Tokárová, A. Pittermannová, J. Čech, P. Ulbrich and F. Štěpánek, Soft Matter, 2012, 8, 1087-1095.

15 M. Ullrich, J. Hanuš, J. Dohnal and F. Štěpánek, J. Colloid Interface Sci., 2013, 394, 380-385.

16 J. Dohnal and F. Štěpánek, Powder Technol., 2010, 200, 254259.

17 G. Pasparakis and N. Bouropoulos, Int. J. Pharm., 2006, 323, 34-42.

18 G. Adam and H. Duncan, Soil Biol. Biochem., 2001, 33, 943951.

19 R. Yao, R. J. Zhang, J. Luan and F. Lin, Biofabrication, 2012, 4, 025007.

20 P. Van Dijken and P. Van Haastert, BMC Cell Biol., 2001, 2, 25. 